\title{
Endoplasmic Reticulum Stress Predicts Clinical Response to Cyclosporine Treatment in Primary Membranous Nephropathy
}

\author{
Jianling Tao ${ }^{a}$ b Wei Zhang ${ }^{a}$ Yubing Wen ${ }^{a}$ Ying Sun ${ }^{a}$ Limeng Chen ${ }^{a}$ Hang Li $^{a}$ \\ Mingxi Li ${ }^{a} \quad$ Xuewang $\mathrm{Li}^{\mathrm{a}}$ Richard A. Lafayette ${ }^{\mathrm{b}}$ Xuemei Li \\ ${ }^{a}$ Division of Nephrology, Peking Union Medical College Hospital, Peking Union Medical College and Chinese Academy of \\ Medical Sciences, Beijing, China; ${ }^{b}$ Division of Nephrology, Department of Medicine, Stanford University School of Medicine, \\ Stanford University, Stanford, Calif., USA
}

\section{Key Words}

Primary membranous nephropathy . Cyclosporine .

Calcineurin . Glucose regulated protein 78 .

Endoplasmic reticulum stress

\begin{abstract}
Background: Little is known about the endoplasmic reticulum stress (ERS) marker glucose regulated protein 78 (GRP78) and calcineurin in the kidney in primary membranous nephropathy (PMN) and if they could predict post-cyclosporine treatment outcome. Methods: This is a retrospective study using a dataset of biopsy-confirmed PMN from Peking Union Medical College Hospital from 1996 to 2014. Seventy-six adult patients treated with cyclosporine as primary immunosuppression for at least 6 months were studied. Immunohistochemistry was used to detect GRP78 and calcineurin in the kidney. Serum calcineurin was assayed by ELISA. Patients were grouped into no-remission (NR, $n=17)$, partial remission (PR, $n=39)$, or complete remission $(C R, n=20)$ at the end of 6 months of treatment. Results: There was no difference of initial dose of cyclosporine among NR, PR, and CR groups. Kidney calcineurin expression in PMN was significantly increased compared to that in controls $(p<0.0083)$. The glomerular GRP78 in NR PMN was higher than that in
\end{abstract}

control, CR and PR patients ( $p<0.0083)$. Kidney calcineurin expression and GRP78 expression was positively correlated. However, there were no differences in either serum calcineurin levels or kidney calcineurin expressions among NR, PR or $\mathrm{CR}$ groups. There was a negative correlation between serum calcineurin activity and whole kidney calcineurin expression $(p=0.034)$ or glomerular calcineurin expression $(p=0.007)$. Neither kidney calcineurin nor GRP78 expression was correlated with proteinuria. Conclusions: ERS marker GRP78 in the glomeruli but not serum or kidney calcineurin expression could be a useful marker in PMN to negatively predict the response to cyclosporine treatment at the sixth month.

(c) 2016 S. Karger AG, Basel

\section{Introduction}

Primary membranous nephropathy $(\mathrm{PMN})$ is a leading cause of nephrotic syndrome in adults. Although 5-30\% of untreated PMN patients achieve spontaneous remission in 5 years, approximately $50 \%$ of patients with PMN develop progressive disease leading to end-stage renal disease requiring dialysis or renal transplantation in 5-10 years [1,2]. KDIGO [3] recommends that cyclosporine or tacrolimus be used for a period of at least 6 months

\section{KARGER}

E-Mail karger@karger.com

www.karger.com/ajn (c) 2016 S. Karger AG, Basel

$0250-8095 / 16 / 0435-0348 \$ 39.50 / 0$
Dr. Jianling Tao, MD

Division of Nephrology, Peking Union Medical College Hospita Peking Union Medical College, Chinese Academy of Medical Sciences Beijing 100730 (China)

E-Mail taojianling@gmail.com 
in PMN patients who meet the criteria for initial therapy, and who choose not to receive the cyclical corticosteroid/ alkylating-agent regimen or who have contraindications to this regimen. Long-term use of cyclosporine may lead to nephrotoxicity including interstitial fibrosis, tubular atrophy and these adverse effects greatly limit its application [4]. Although complete and partial remission (PR) in proteinuria was seen in approximately $2 / 3$ of the patients [2], it would be ideal that potential non responders should be selected to avoid such therapy and given other options. We were thus interested to find new biomarkers to predict clinical response of PMN patients to cyclosporine therapy prior to treatment, to prevent unnecessary cyclosporine exposure and avoid potentially severe side effects in non-responders.

Calcineurin is a $\mathrm{Ca}^{2+}$-/calmodulin-dependent serine/ threonine protein phosphatase and serves as a target of calcineurin inhibitors (CNIs), cyclosporine and tacrolimus [5]. CNIs induce complete or PR through immunologic and non-immunologic ways. Previous study has shown that cyclosporine effects and toxicity depend on calcineurin and cyclophilin distribution in tissues [6]. Whether calcineurin expression in the kidney is related to clinical response to cyclosporine in PMN patients remains questionable. This study is to investigate if renal calcineurin is a determinant of the clinical response to cyclosporine treatment [7].

Endoplasmic reticulum (ER) is an organelle for protein folding, assembling and maturation. ER function is vulnerable to various pathophysiological effects such as hypoxia, $\mathrm{Ca}^{2+}$ depletion, viral infections and oxidative stress. These effects lead to the activation of the unfolded protein response (UPR), a protective response to accumulation of misfolded proteins in the ER, namely, ER stress (ERS) [8]. Glucose regulated protein 78 (GRP78), also known as 'immunoglobulin heavy chain-binding protein' (BiP), a member of the heat-shock protein-70 family, is involved in the folding and assembly of proteins in the ER [9]. As a major ER chaperone, GRP78 is antiapoptotic and protects the host cell against ERS-induced cell death $[10,11]$. Evidence shows that increased proteinuria can induce ERS in renal proximal tubule cells [12-14] and podocytes [15]. Our previous in vitro study has also shown increased expression of calcineurin in podocytes during palmitate-induced ERS, thus linking these 2 markers [16].

It has also been suggested that ERS is a mediator of cyclosporine nephrotoxicity $[17,18]$. From a clinical perspective, no study had been done about status of ERS or calcineurin in the kidney of PMN. In this study, we tried to explore if serum calcineurin, renal calcineurin, or renal GRP78 expression, as a marker of ERS, was correlated with patients' clinical responses to cyclosporine treatment.

\section{Methods}

\section{Patients and Data Collection}

Seventy-six patients at Peking Union Medical College Hospital (from January 1996 to June 2014), who had newly diagnosed, biopsy-proven PMN and were treated with cyclosporine as primary immunosuppression for at least 6 months, were retrospectively reviewed. Kidney biopsies were evaluated by light microscopy, electron microscopy and immunofluorescence microscopy. Secondary causes were carefully excluded to confirm PMN. Subjects with previous cyclophosphamide or other immunosuppressive agent treatment were excluded. Kidney samples (away from the tumor) from 5 renal carcinoma patients in the Division of Urology were used as healthy controls in staining. Serum from IgA nephropathy (IgAN, $\mathrm{n}=12)$, lupus nephritis $(\mathrm{LN}, \mathrm{n}=12)$, minimal change disease (MCD, $n=12)$, and PMN $(n=42)$ without any immunosuppressant exposure during the past 6 months before serum collection were assayed for serum calcineurin activity. Serums from 14 healthy subjects during their regular physical checkup were used as controls in the serum calcineurin assay. Kidney biopsy tissues of all PMN patients $(n=76)$ were used for calcineurin and GRP78 immunohistochemistry (IHC) staining.

\section{Grouping}

PMN patients were divided into 3 groups at the end of 6 months of cyclosporine treatment based on their clinical response. Complete remission (CR) was defined as urinary protein $<0.30 \mathrm{~g} /$ day and serum albumin $>35 \mathrm{~g} / \mathrm{l}$; PR was defined as urinary protein excretion between 0.3 and $3.5 \mathrm{~g} / \mathrm{day}$, with a decrease in proteinuria by of at least $50 \%$ from the initial value and $<3.5 \mathrm{~g} /$ day; accompanied by an improvement or normalization of the serum albumin concentration and stable serum creatinine (Scr) [3]. Patients who did not meet the definitions of $\mathrm{CR}$ or PR were assigned to no-remission (NR).

\section{Kidney IHC of Calcineurin and GRP78}

Kidney tissues were fixed with $10 \%$ formaldehyde solution, embedded in paraffin and cut into $2-\mu \mathrm{m}$ thick sections. After deparaffinization, hydration and antigen retrieval, the tissue samples were incubated with antibody at a dilution of 1:2,000 against calcineurin (Abcam, ab71149, UK) at $4^{\circ} \mathrm{C}$ overnight or a dilution of $1: 100$ of an antibody against GRP78 (Santa Cruz, sc-1050, USA) at $37^{\circ} \mathrm{C}$ for $1 \mathrm{~h}$. In staining of calcineurin, antigen retrieval was performed by heat-induced retrieval by keeping slides at a temperature of $120^{\circ} \mathrm{C}$ in a pressure cooker for $3 \mathrm{~min}$ in the solution of Tris/EDTA buffer ( $\mathrm{pH}$ 9.0). In staining of GRP78, antigen retrieval was performed by protease-induced retrieval by incubating slides with a solution of $0.1 \%$ trypsin at a temperature of $60^{\circ} \mathrm{C}$ for $20 \mathrm{~min}$.

After blocking endogenous peroxidase with $3 \%$ hydrogen peroxide for $20 \mathrm{~min}$, samples were then incubated with biotin-labeled secondary antibody at $37^{\circ} \mathrm{C}$ for $1 \mathrm{~h}$. Slides were then exposed to color development by diaminobenzidine. Images were semi-quan- 
Table 1. Baseline characteristics of 76 adult patients with IMN treated with cyclosporine

\begin{tabular}{lcccc}
\hline & CR $(\mathrm{n}=20)$ & PR $(\mathrm{n}=39)$ & NR $(\mathrm{n}=17)$ & $\mathrm{p}$ value \\
\hline Age, years & $46.4 \pm 16.5(20)$ & $47.9 \pm 15.8(39)$ & $53.0 \pm 14.4(17)$ & 0.411 \\
Gender, male/female & $10 / 10$ & $26 / 13$ & $15 / 2$ & 0.047 \\
SBP, mm Hg & $126.4 \pm 16.5(19)$ & $125.5 \pm 17.2(33)$ & $134.4 \pm 20.7(12)$ & 0.263 \\
DBP, mm Hg & $76.1 \pm 9.5(19)$ & $75.6 \pm 11.4(33)$ & $81.8 \pm 9.8(15)$ & 0.159 \\
Proteinuria, g/day & $3.6 \pm 3.4(20)$ & $5.6 \pm 3.4^{\wedge}(39)$ & $10.3 \pm 6.2^{*}(17)$ & $<0.001$ \\
Serum albumin, g/l & $28.8 \pm 4.3(20)$ & $24.8 \pm 5.0^{\wedge}(39)$ & $22.0 \pm 4.7^{*}(17)$ & $<0.001$ \\
Scr, $\mu$ mol/l & $69.3 \pm 13.1(20)$ & $74.7 \pm 18.5(39)$ & $84.8 \pm 16.6^{*}(17)$ & 0.022 \\
CsA dose, mg/kg/day & $2.9 \pm 1.1(20)$ & $2.7 \pm 0.8(39)$ & $3.0 \pm 1.0(17)$ & 0.49 \\
Prednisone dose, mg/kg/day & $0.55 \pm 0.15(18)$ & $0.46 \pm 0.20(33)$ & $0.45 \pm 0.21(13)$ & 0.169 \\
\hline
\end{tabular}

$\mathrm{n}=$ The number of patients studied. SBP = Systolic blood pressure; DBP = diastolic blood pressure; CsA = cyclosporine A.

* Compared to CR or PR group; ^ compared to CR group.

titatively analyzed by Image-Pro Plus 6 software. At least 5 images were captured at random in each slide of kidney tissue for quantification. Calcineurin and GRP78 expression were assessed by the integrated optical density (OD) of the color object above a baseline threshold.

\section{Serum Calcineurin Activity Assay}

Serum calcineurin activity was measured by a colorimetric assay kit (calcineurin phosphatase assay kit, Enzo Life Sciences, BML-AK804, USA). This method is based on the principle that calcineurin can dephosphorylate the RII phosphopeptide substrate. The detection of released free-phosphate is based on the classic malachite green assay. The standard curve was made by serial dilution of phosphate standard and its corresponding OD 620 $\mathrm{nm}$ value on a microplate reader. The OD values were converted to the amount of released free-phosphate using a standard curve formula, which indirectly indicates calcineurin activity.

\section{Statistical Analysis}

All data analyses were performed using SPSS version 17.0. For continuous measurements, if they were normally distributed, they were expressed as mean \pm SD and one-way analysis of variance was used within group comparisons. If continuous data were not normally distributed, they were presented by median (IQR), and non-parametric test Mann-Whitney U test was applied within group comparisons. In order to control the type I error, Bonferroni's correction was made for all possible comparisons, according to the number between any 2 subgroups being compared among the total groups, $\mathrm{p}<0.0083(0.05 / 6)$ was considered statistically significant for serum calcineurin, renal calcineurin expression, and renal GRP78 expression comparisons between control and sub-groups in PMN, and $\mathrm{p}<0.005(0.05 / 10)$ was considered statistically significant for comparisons of serum calcineurin activity among control, IgAN, MCD, LN and PMN sub-groups.

For normally distributed data, Pearson's correlation analysis was used. For non-normally distributed data, Spearman correlation analysis was applied. $\mathrm{p}<0.05$ was considered significant unless otherwise indicated.

\section{Results}

\section{Baseline Characteristics of Patients Enrolled in This Study}

Baseline data for the 76 patients were listed in table 1. There was no difference in the initial cyclosporine dose among the 3 subgroups. Proteinuria in the NR group was significantly higher than those in the CR or PR groups $(\mathrm{p}<0.001)$. Correspondingly, the serum albumin level was significantly lower in the NR group than those in the CR or PR group ( $\mathrm{p}<0.001)$. Scr level was also significantly higher in the NR group than in the CR or PR group $(\mathrm{p}=$ 0.022 ). A total of 64 patients were treated with prednisone at the same time, and the mean daily dose was $0.48 \mathrm{mg} /$ $\mathrm{kg} /$ day, with no difference among these 3 subgroups ( $\mathrm{p}=$ 0.169). After 6 months of cyclosporine therapy, there were 20,39, and 17 patients respectively in CR, PR and NR groups. The overall complete and $\mathrm{PR}$ rate was about $77 \%$. The proteinuria, serum albumin level, Scr level, the cyclosporine and prednisone doses were listed in table 2. Patients in NR and PR groups maintained higher cyclosporine dosage as compared to the CR group $(p=0.009)$.

\section{Calcineurin and GRP78 Expression in the Kidney and} Their Correlation

IHC demonstrated that calcineurin was mainly expressed in podocytes and distal tubules (fig. 1a-d). Calcineurin staining was increased in $\mathrm{PMN}$ patients compared to $\mathrm{HC}$ before cyclosporine therapy in the glomerular and tubular compartments. Although no significant differences were observed among CR, PR, and NR subgroups in either the glomerular or tubular compartment (fig. 1e, $\mathrm{f}$ and $\mathrm{g}$ ), there was a clear trend that non-responders to 
Table 2. Clinical profile of 76 adult patients with IMN treated with cyclosporine after 6 months

\begin{tabular}{llllr}
\hline & CR $(\mathrm{n}=20)$ & PR $(\mathrm{n}=39)$ & NR $(\mathrm{n}=17)$ & p value \\
\hline Proteinuria, g/day & $0.14 \pm 0.10(20)$ & $0.82 \pm 0.85^{\#}(39)$ & $5.11 \pm 4.24^{*}(17)$ & $<0.001$ \\
Serum albumin, g/l & $42.0 \pm 3.8(20)$ & $40.0 \pm 6.0(39)$ & $30.0 \pm 9.5^{*}(17)$ & $<0.001$ \\
Scr, $\mu \mathrm{mol} / \mathrm{l}$ & $79.0 \pm 33.1(20)$ & $82.0 \pm 23.0(39)$ & $88.0 \pm 32.1(17)$ & 0.359 \\
CsA dose, mg/kg/day & $1.69 \pm 0.91(20)$ & $2.44 \pm 0.81^{\#}(39)$ & $2.31 \pm 0.98^{\#}(17)$ & 0.009 \\
Prednisone dose, mg/kg/day & $0.31 \pm 0.20(9)$ & $0.31 \pm 0.14(16)$ & $0.41 \pm 0.06(6)$ & 0.392 \\
\hline
\end{tabular}

$\mathrm{n}=$ The number of patients studied. CsA = Cyclosporine A.

* Compared to CR or PR group; ${ }^{\#}$ compared to CR group.



Fig. 1. Representative images of calcineurin stain in the kidneys from healthy control and IMN patients (IHC, $\times 200$ ), and its quantitative analysis. a Peri tissue from dissected renal cancer; $\mathbf{b} \mathrm{CR}$; c PR; d NR; e calcineurin quantification in the whole kidney; $\mathbf{f}$ cal-

cyclosporine had a higher renal calcineurin expression than responders.

IHC demonstrated that GRP78 was mainly expressed in the cytoplasm of podocytes and distal tubules (fig. 2a-d). The GRP78 in the whole kidney was significantly higher in NR group than in the CR or PR subgroup (fig. 2e). The glomerular GRP78 in the PMN NR group was higher than that of healthy control, CR or PR subgroup (fig. 2f). The tubulointerstitial GRP78 in the NR group of PMN was also higher than that in the CR or PR subgroup (fig. $2 \mathrm{~g}$ ). cineurin expression quantification in the glomeruli; $\mathbf{g}$ calcineurin expression quantification in the tubulointerstitial compartment. CAN $=$ Calcineurin. ${ }^{*} \mathrm{p}<0.0083$ vs. HC. HC: $\mathrm{n}=5$, CR: $\mathrm{n}=20$, PR: $\mathrm{n}=39$, NR: $\mathrm{n}=17$.

There was a significant positive correlation between calcineurin and GRP78 in overall expression $(\mathrm{p}=0.025$; fig. 3a), in the glomerular compartment $(\mathrm{p}<0.001$; fig. $3 \mathrm{~b})$ and in the tubular interstitial compartment $(\mathrm{p}=0.025$; fig. 3c). There was no correlation between whole kidney, glomerular or tubular interstitial calcineurin or GRP78 expression with baseline proteinuria (fig. $3 \mathrm{~d}-\mathrm{i}$ ). There was no correlation between whole kidney, glomerular or tubular interstitial calcineurin or GRP78 expression with baseline serum albumin level or Scr level, either (data not shown). 


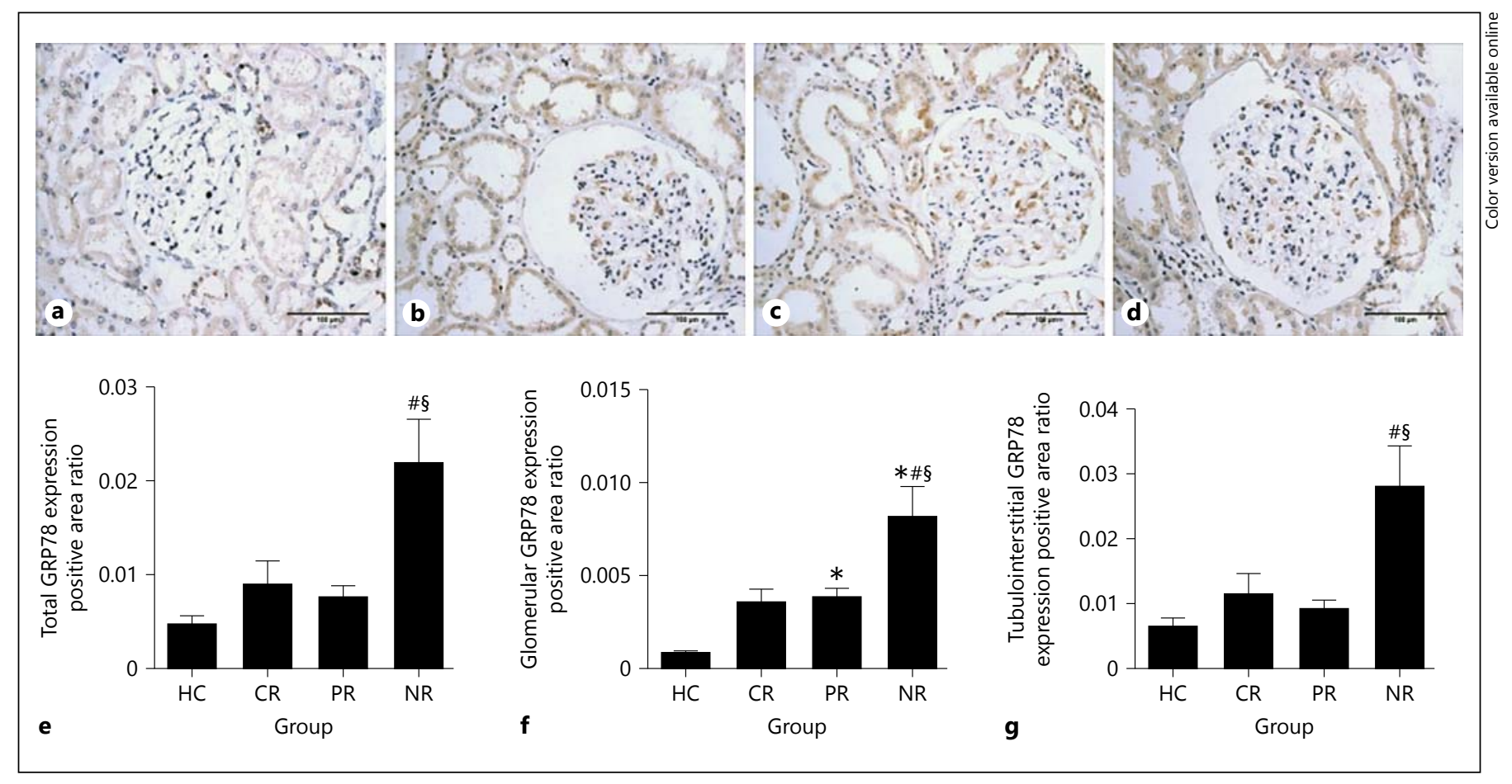

Fig. 2. Representative images of GRP78 stain in the kidneys of healthy control and IMN (IHC, $\times 200)$, and its quantitative analysis. a Peri tissue from dissected renal cancer; b CR; c PR; d NR; e GRP78 quantification in the whole kidney; f GRP78 quantification in the glomeruli; g GRP78 quantification in the tubulointerstitial compartment. ${ }^{*} \mathrm{p}<0.0083$ vs. HC; ${ }^{\#} \mathrm{p}<0.0083$ vs. CR; ${ }^{\S} \mathrm{p}<0.0083$ vs. PR. HC: $n=5$, CR: $n=20$, PR: $n=39$, NR: $n=17$.

\section{Discussion}

\section{Levels of Calcineurin Activity in Serum and Its}

Correlation with Renal Calcineurin Expression

Serum calcineurin activity in $P M N$ patients $(n=42)$ was significantly higher than $\mathrm{HC}(\mathrm{n}=14)$, but there were no differences among CR, PR and NR subgroups (fig. 4a). In order to verify whether high serum calcineurin expression is unique in PMN, sera from IgAN, LN, and MCD patients ( $\mathrm{n}=12$ in each group) were analyzed. The characteristics of those patients are listed in table 3. PMN, LN and MCD patients had higher calcineurin activity than $\mathrm{HC}$ and IgAN patients (fig. 4b). PMN patients also had significantly higher calcineurin activity than LN and MCD patients. A total of $37 \mathrm{PMN}$ patients in this cohort had both renal tissues evaluated by IHC for calcineurin and serum assayed for its activity. There was a negative correlation between serum calcineurin activity with the whole renal calcineurin expression ( $\mathrm{p}=0.034$; fig. $4 \mathrm{c}$ ). There was a negative correlation between serum calcineurin activity with the glomerular calcineurin expression ( $\mathrm{p}=0.007$; fig. $4 \mathrm{~d})$. There was no correlation between serum calcineurin activity with the tubulointerstitial calcineurin expression (fig. 4e). There was also no correlation between serum calcineurin activity and baseline proteinuria in PMN patients (fig. $4 \mathrm{f}$ ).
PMN is the most common nephrotic syndrome in adults in western country and China. In a meta-analysis, Xie et al. [19] included 17 randomized controlled trials encompassing 696 Chinese PMN patients, who had been treated with different immunosuppressive agents, where 7 trials involving 282 patients in comparison CNIs with alkylating agents, found that CNIs led to a higher effect on CR and PR rate with fewer side effects in the Chinese population. While studies have demonstrated efficacy of CNIs, safety concerns include multiple side effects, including the risk of nephrotoxicity with long-term exposure [20]. This makes it very attractive to find a biomarker predictive of the clinical response to CNIs [21].

ERS is defined by an accumulation of unfolded or misfolded proteins in the ER, both physiologically and pathologically. In response to this accumulation of unfolded/ misfolded proteins, cells adapt to the stressful conditions through a UPR [22]. The UPR involves transient attenuation of new protein synthesis, induction of ER chaperones, such as GRP78, and activation of ER-associated degradation to eliminate immature proteins [23]. 




Fig. 3. Correlation analysis among GRP78, calcineurin and proteinuria. Between the whole kidney calcineurin and the whole GRP78 expression (a), between glomerular calcineurin and GRP78 expression (b), between interstitial calcineurin and GRP78 expression (c), between the whole kidney calcineurin expression with proteinuria (d), between glomerular calcineurin expression with

Accumulating evidence indicates that ERS contributes to glomerular and tubular injury in kidney disease. These findings emphasize the importance of ERS as a not yet unidentified pathway in its pathogenesis and the potential possibility of reno-protective strategies targeting ERS [24, 25]. Surprisingly, studies specific for GRP78 expression in proteinuria (e), between interstitial calcineurin expression with proteinuria (f), between the whole kidney GRP78 expression with proteinuria (g), between glomerular GRP78 expression with proteinuria (h), between interstitial GRP78 expression with proteinuria (i).

glomerular disease are lacking. There is some evidence showing that GRP78 protein levels are increased in PMN compared with MCD in biopsied human kidney samples [23]. This raised another question: how does GRP78 expression change in the renal histology of PMN patients, and can this be a useful marker to predict patients' clinical 


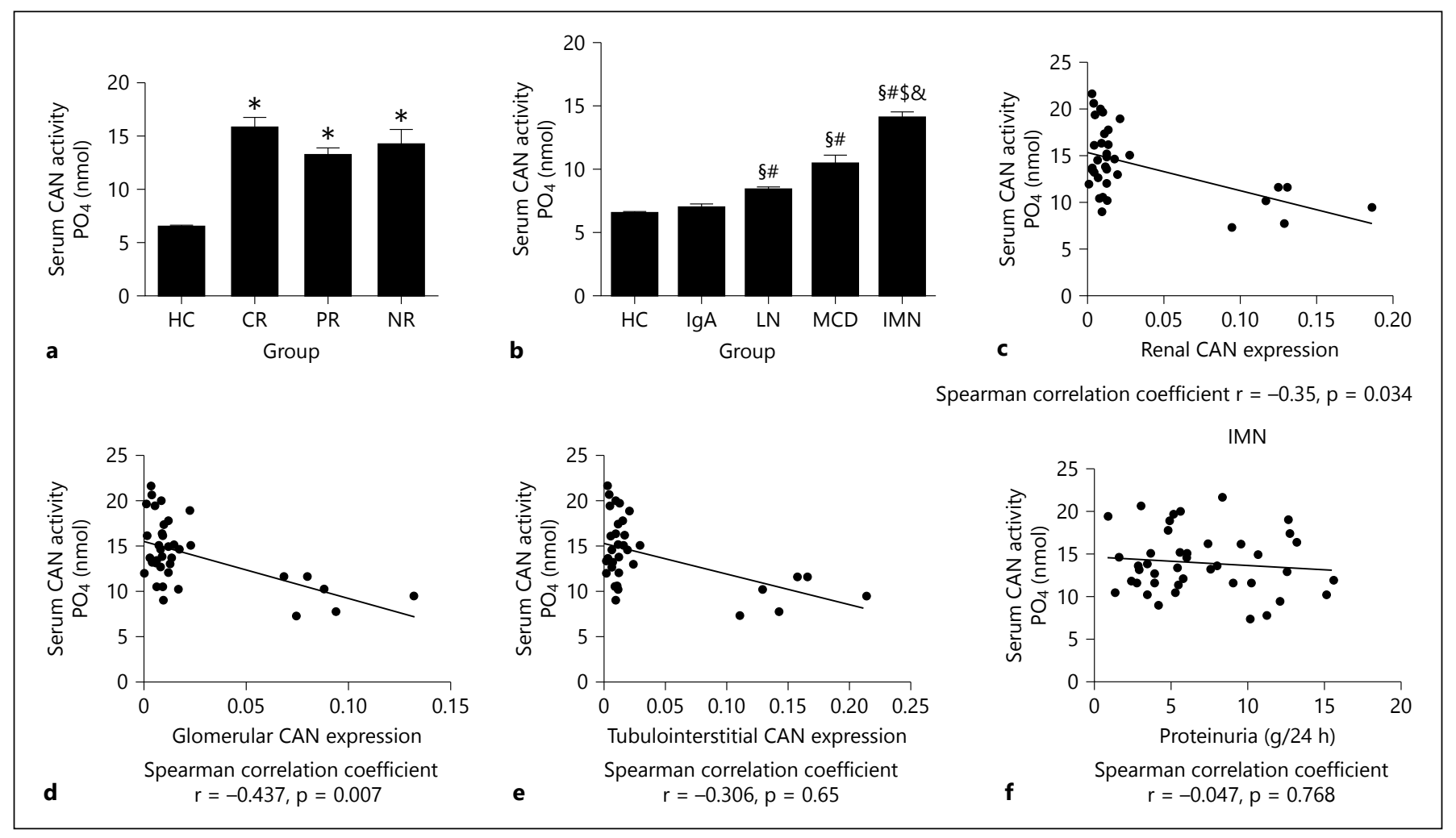

Fig. 4. Serum calcineurin activity in patients with $P M N($ a), IgAN, $\mathrm{LN}$, and MCD (b). In PMN patients, there is a negative correlation between serum calcineurin activity and total renal calcineurin expression (c), and with glomerular calcineurin expression (d). The correlation analysis between serum calcineurin activity and tubule interstitial calcineurin expression (e). The correlation analysis between serum calcineurin activity and proteinuria in $\mathrm{PMN}$ patients (f). ${ }^{*} \mathrm{p}<0.0083$ vs. HC; ${ }^{\#} \mathrm{p}<0.005$ vs. IgA; ${ }^{\S} \mathrm{p}<0.005$ vs. LN; ${ }^{\&} \mathrm{p}<$ 0.005 vs. MCD.

Table 3. Clinical characteristics of patients from IgAN, MCD, LN, and PMN with serum calcineurin activity assayed

\begin{tabular}{lccccr}
\hline & IgA $(\mathrm{n}=12)$ & MCD $(\mathrm{n}=12)$ & LN $(\mathrm{n}=12)$ & IMN $(\mathrm{n}=42)$ & p value \\
\hline Age, years & $37.8 \pm 14.3$ & $28.8 \pm 14$ & $34.3 \pm 14$ & $47.9 \pm 16.4^{\#}$ & $<0.002$ \\
Gender, male/female & $10 / 2$ & $10 / 2$ & $3 / 9$ & $29 / 13$ & 0.008 \\
Serum albumin, g/l & $36.4 \pm 6.4$ & $20.6 \pm 5.7^{*}$ & $25.2 \pm 9.5^{*}$ & $24.8 \pm 4.9^{*}$ & $<0.006$ \\
Scr, $\mu$ mol/l & $177.9 \pm 116.7$ & $104.4 \pm 83.3$ & $128.2 \pm 110.3$ & $77.0 \pm 18.1^{*}$ & $<0.001$ \\
Proteinuria, g/24h & $3.8 \pm 3.4$ & $11.3 \pm 10.1^{*}$ & $9.7 \pm 8.9^{*}$ & $6.8 \pm 4.0$ & 0.016 \\
\hline
\end{tabular}

* Compared to the IgAN group; ${ }^{\#}$ compared to the MCD group.

responses to cyclosporine treatment? If it were, we could predictively shift those patients who will not respond to cyclosporine treatment to other treatments and avoid unnecessary potential CNI nephrotoxicity [26].

CNIs exert their immunosuppressive effect by blocking T-cell-mediated responses through the inhibition of calcineurin-dependent nuclear factor of activated T-cells signaling [27]. CNIs have also been suggested to be cytoprotective in podocytes through the inhibition of synaptopodin degradation and stabilization of the podocyte actin cytoskeleton [28]. Cyclosporine has also been shown to induce stiffening of the actin network in tubu- 
lar structure, which may alter the response of proximal cells to modifications of tubular flow, potentially exerting a detrimental effect on tubular structure [29]. The physiologic role of calcineurin in kidney cells had largely remained unknown until the exhibition of abnormal kidney development in mice with knockouts for the calcineurin Aa subunit [30]. However, the distribution pattern of calcineurin in the kidney histology and its correlation to response to CNIs treatment have not been studied.

Interestingly, Pallet et al. [17] found that cyclosporine can induce tubular phenotypic changes and death through ERS. We were also interested in the relation between ERS and calcineurin in the kidneys of idiopathic membranous nephropathy (IMN) patients, hoping to facilitate our understanding of the pathogenesis of proteinuria in PMN.

In this large PMN cohort $(n=76)$, we have shown that both calcineurin and GRP78 are mainly expressed in the podocytes and distal tubules in patients with PMN. Kidney calcineurin expression is upregulated in PMN patients, both in glomerular and in tubular sections. Unexpectedly, while an increasing trend in kidney calcineurin expression was observed when patients were less responsive to cyclosporine, there were no significant differences among groups based on the patients' clinical response to cyclosporine treatment. Further validation of this trend to be truly clinically significant or not is needed in a larger sample of study.

Also, we demonstrate that GRP78, an ERS marker, is significantly increased in the kidney of PMN patients who will not respond to cyclosporine treatment. These findings suggest that ERS may be involved in the pathogenesis of PMN, such hypothesized as ERS could aggravate proteinuria, which is an independent clinical predictor of clinical outcome. They further suggest that GRP78 expression in glomeruli can be a useful marker before patients with PMN are considered for a course of cyclosporine treatment. We are not able to provide a confirmed cutoff value with a numeric level, above which the patient is supposed to be non-responsive to the cyclosporine therapy. However, intensity of kidney ERS may serve as a significant sign for clinicians in their clinical judgment of whether the case will be treated by cyclosporine successfully or not.

Finally, this study is also the first to assay the serum calcineurin activity in patients with glomerular disease such as PMN, IgAN, MCD, and LN. Sankaranarayanan et al. [31] have previously shown an inverse correlation between serum calcineurin activity and glycosylated he- moglobin levels in diabetic patients, suggesting that serum calcineurin activity may be useful in the simultaneous assessment of oxidative stress and glycemic control in type II diabetes mellitus. It is interesting that PMN patients have the highest level of serum calcineruin activity compared to IgAN, MCD, and LN. The observed inverse relationship between serum calcineurin activity and renal calcineurin expression, and its significance in the role of calcineurin in the kidney remains to be explored further.

Even though it has been shown that ERS is an important pathway in proteinuric kidney diseases [32], its mechanism is not fully understood. Calcineurin is known to possess numerous functions, such as cAMP metabolism, microtubule assembly, skeletal muscle metabolism, T-cell activation, and cardiac hypertrophy [33]. Proteinuria is a strong independent clinical predictor in PMN outcome [34]. In this study, we demonstrate that there is a significant correlation between kidney calcineurin expression and GRP78 expression. However, neither kidney ERS nor calcineurin expression is correlated with proteinuria. While the study does not address the causeeffector role between GRP78 and calcineurin, or its crosstalk in the sophisticated biological network, it does provide a target about future research on the pathophysiological role of this connection in PMN.

Despite its retrospective nature, we think that this large cohort human study in PMN patients will outweigh its limitations. We provide important information regarding the expression of calcineurin and ER in the kidney and find they were correlated to each other in PMN. We also suggest that glomerular GRP78 expression level before cyclosporine treatment, instead of serum or kidney calcineurin levels can be a useful marker to predict an individual's clinical response to cyclosporine. This focus on the relevance of renal ERS status in the non-responders to cyclosporine treatment may shed light on further development of medications targeting ERS for non-responders to cyclosporine treatment.

\section{Acknowledgments}

The work was supported by the National Natural Science Foundation of China (No. 81170665) to Dr. J. Tao.

\section{Disclosure Statement}

The authors do not have any competing interests. 


\section{References}

- Schieppati A, Mosconi L, Perna A, Mecca G, Bertani T, Garattini S, Remuzzi G: Prognosis of untreated patients with idiopathic membranous nephropathy. N Engl J Med 1993; 329:85-89.

-2 Cattran DC, Alexopoulos E, Heering P, Hoyer PF, Johnston A, Meyrier A, Ponticelli C, Saito T, Choukroun G, Nachman P, Praga M, Yoshikawa N: Cyclosporin in idiopathic glomerular disease associated with the nephrotic syndrome: workshop recommendations. Kidney Int 2007;72:1429-1447.

-3 Radhakrishnan J, Cattran DC: The KDIGO practice guideline on glomerulonephritis: reading between the (guide)lines - application to the individual patient. Kidney Int 2012;82:840-856.

-4 Chapman JR, Nankivell BJ: Nephrotoxicity of ciclosporin A: short-term gain, long-term pain? Nephrol Dial Transplant 2006;21:2060 2063.

5 Wakamatsu A, Fukusumi Y, Hasegawa E, Tomita M, Watanabe T, Narita I, Kawachi $\mathrm{H}$ : Role of calcineurin $(\mathrm{CN})$ in kidney glomerular podocyte: $\mathrm{CN}$ inhibitor ameliorated proteinuria by inhibiting the redistribution of $\mathrm{CN}$ at the slit diaphragm. Physiol Rep 2016; 4:pii:e12679.

6 Kung L, Batiuk TD, Palomo-Pinon S, Noujaim J, Helms LM, Halloran PF: Tissue distribution of calcineurin and its sensitivity to inhibition by cyclosporine. Am J Transplant 2001;1:325-333.

7 Naesens M, Kuypers DR, Sarwal M: Calcineurin inhibitor nephrotoxicity. Clin J Am Soc Nephrol 2009;4:481-508.

8 Taniguchi M, Yoshida H: Endoplasmic reticulum stress in kidney function and disease. Curr Opin Nephrol Hypertens 2015;24:345350.

9 Hendershot LM, Valentine VA, Lee AS, Morris SW, Shapiro DN: Localization of the gene encoding human $\mathrm{BiP} / \mathrm{GRP78}$, the endoplasmic reticulum cognate of the HSP70 family, to chromosome 9q34. Genomics 1994;20:281284.

10 Wang M, Wey S, Zhang Y, Ye R, Lee AS: Role of the unfolded protein response regulator GRP78/BiP in development, cancer, and neurological disorders. Antioxid Redox Signal 2009;11:2307-2316.

-11 Rao RV, Peel A, Logvinova A, del Rio G, Hermel E, Yokota T, Goldsmith PC, Ellerby LM, Ellerby HM, Bredesen DE: Coupling endoplasmic reticulum stress to the cell death program: role of the ER chaperone GRP78. FEBS Lett 2002;514:122-128.
12 Ohse T, Inagi R, Tanaka T, Ota T, Miyata T, Kojima I, Ingelfinger JR, Ogawa S, Fujita T, Nangaku M: Albumin induces endoplasmic reticulum stress and apoptosis in renal proximal tubular cells. Kidney Int 2006;70:14471455.

13 Fang L, Xie D, Wu X, Cao H, Su W, Yang J: Involvement of endoplasmic reticulum stress in albuminuria induced inflammasome activation in renal proximal tubular cells. PLoS One 2013;8:e72344.

14 Gorostizaga A, Mori Sequeiros Garcia MM, Acquier A, Gomez NV, Maloberti PM, Mendez CF, Paz C: Modulation of albumin-induced endoplasmic reticulum stress in renal proximal tubule cells by upregulation of MAPK phosphatase-1. Chem Biol Interact 2013;206:47-54.

15 Chen S, He FF, Wang H, Fang Z, Shao N, Tian XJ, Liu JS, Zhu ZH, Wang YM, Wang S, Huang K, Zhang C: Calcium entry via TRPC6 mediates albumin overload-induced endoplasmic reticulum stress and apoptosis in podocytes. Cell Calcium 2011;50:523-529.

16 Hu RR, Ma J, Tao JL, Wen YB, Zhao C, Zhang W, Li H, Li MX, Li XM, Li XW: [Expression of calcineurin in podocytes in response to endoplasmic reticulum stress in diabetic nephropathy]. Zhongguo Yi Xue Ke Xue Yuan Xue Bao 2014;36:477-481.

17 Pallet N, Bouvier N, Bendjallabah A, Rabant M, Flinois JP, Hertig A, Legendre C, Beaune P, Thervet E, Anglicheau D: Cyclosporine-induced endoplasmic reticulum stress triggers tubular phenotypic changes and death. Am J Transplant 2008;8:2283-2296.

18 Sarro E, Jacobs-Cachá C, Itarte E, Meseguer A: A pharmacologically-based array to identify targets of cyclosporine A-induced toxicity in cultured renal proximal tubule cells. Toxicol Appl Pharmacol 2012;258:275-287.

19 Xie G, Xu J, Ye C, Chen D, Xu C, Yang L, Ma Y, Hu X, Li L, Sun L, Zhao X, Mao Z, Mei C: Immunosuppressive treatment for nephrotic idiopathic membranous nephropathy: a meta-analysis based on Chinese adults. PLoS One 2012;7:e44330.

20 Cattran DC, Appel GB, Hebert LA, Hunsicker LG, Pohl MA, Hoy WE, Maxwell DR, Kunis CL; North America Nephrotic Syndrome Study Group: Cyclosporine in patients with steroid-resistant membranous nephropathy: a randomized trial. Kidney Int 2001;59:14841490.

21 Tran TH, Hughes GJ, Greenfeld C, Pham JT: Overview of current and alternative therapies for idiopathic membranous nephropathy. Pharmacotherapy 2015;35:396-411.
22 Yoshida H: ER stress and diseases. FEBS J 2007;274:630-658

23 Markan S, Kohli HS, Joshi K, Minz RW, Sud K, Ahuja M, Anand S, Khullar M: Up regulation of the GRP-78 and GADD-153 and down regulation of Bcl-2 proteins in primary glomerular diseases: a possible involvement of the ER stress pathway in glomerulonephritis. Mol Cell Biochem 2009;324:131-138.

24 Inagi R: Endoplasmic reticulum stress as a progression factor for kidney injury. Curr Opin Pharmacol 2010;10:156-165.

25 Cybulsky AV: The intersecting roles of endoplasmic reticulum stress, ubiquitin-proteasome system, and autophagy in the pathogenesis of proteinuric kidney disease. Kidney Int 2013;84:25-33.

26 Fervenza FC: Overcoming calcineurin dependence in membranous nephropathy: is rituximab the answer? Clin J Am Soc Nephrol 2009;4:1017-1019.

27 Zhang B, Shi W: Is the antiproteinuric effect of cyclosporine A independent of its immunosuppressive function in $\mathrm{T}$ cells? Int $\mathrm{J}$ Nephrol 2012;2012:809456.

28 Faul C, Donnelly M, Merscher-Gomez S, Chang YH, Franz S, Delfgaauw J, Chang JM, Choi HY, Campbell KN, Kim K, Reiser J, Mundel P: The actin cytoskeleton of kidney podocytes is a direct target of the antiproteinuric effect of cyclosporine A. Nat Med 2008; 14:931-938.

29 Descazeaud V, Mestre E, Marquet P, Essig M: Calcineurin regulation of cytoskeleton organization: a new paradigm to analyse the effects of calcineurin inhibitors on the kidney. J Cell Mol Med 2012;16:218-227.

30 Gooch JL, Toro JJ, Guler RL, Barnes JL: Calcineurin A-alpha but not A-beta is required for normal kidney development and function. Am J Pathol 2004;165:1755-1765.

31 Sankaranarayanan TK, Sethi BK, Subramanyam C: Serum calcineurin activity in relation to oxidative stress and glycemic control in type II diabetes mellitus. Clin Biochem 2005; 38:218-222.

32 Cybulsky AV: Endoplasmic reticulum stress in proteinuric kidney disease. Kidney Int 2010;77:187-193.

33 Kurji K, Sharma RK: Potential role of calcineurin in pathogenic conditions. Mol Cell Biochem 2010;338:133-141.

34 McQuarrie EP, Stirling CM, Geddes CC: Idiopathic membranous nephropathy and nephrotic syndrome: outcome in the era of evidence-based therapy. Nephrol Dial Transplant 2012;27:235-242. 


\section{Erratum}

\section{Nephrology}

In the article by Tao J, et al., entitled 'Endoplasmic reticulum stress predicts clinical response to cyclosporine treatment in primary membranous nephropathy' [Am J Nephrol 2016;43:348-356, DOI: 10.1159/000446293], the following 2 errors occurred in table 2: it should be $0.82(0.85)$ instead of $0.82 \pm 0.85$ and similarly 5.11 (4.24) instead of 5.11 \pm 4.24 .

Table 2. Clinical profile of 76 adult patients with IMN treated with cyclosporine after 6 months

\begin{tabular}{llllr}
\hline & CR $(\mathrm{n}=20)$ & PR $(\mathrm{n}=39)$ & NR $(\mathrm{n}=17)$ & p value \\
\hline Proteinuria, g/day & $0.14 \pm 0.10(20)$ & $0.82(0.85)^{\#}(39)$ & $5.11(4.24)^{*}(17)$ & $<0.001$ \\
Serum albumin, g/l & $42.0 \pm 3.8(20)$ & $40.0 \pm 6.0(39)$ & $30.0 \pm 9.5^{*}(17)$ & $<0.001$ \\
Scr, $\mu$ mol/l & $79.0 \pm 33.1(20)$ & $82.0 \pm 23.0(39)$ & $88.0 \pm 32.1(17)$ & 0.359 \\
CsA dose, $\mathrm{mg} / \mathrm{kg} /$ day & $1.69 \pm 0.91(20)$ & $2.44 \pm 0.81^{\#}(39)$ & $2.31 \pm 0.98^{\#}(17)$ & 0.009 \\
Prednisone dose, mg/kg/day & $0.31 \pm 0.20(9)$ & $0.31 \pm 0.14(16)$ & $0.41 \pm 0.06(6)$ & 0.392 \\
\hline
\end{tabular}

$\mathrm{n}=$ The number of patients studied. CsA = Cyclosporine A.

* Compared to CR or PR group; " compared to CR group. 